\title{
Rehabilitation Environmental System Design of children with autism from the analysis of visual perception
}

\author{
Li Deng ${ }^{a}$, Li Li ${ }^{\text {b, }}$, Yuyan Mao ${ }^{\mathrm{c}}$ \\ Wuhan Polytechnic University, Wuhan Hubei, 430023 \\ a dengli316@sohu.com, ${ }^{\text {b. }}$ Corresponding author Email: lilywh@whpu.edu.cn, \\ cymy920707@163.com
}

Keywords: children with autism; ergonomics; healing environment; visual system design;

\begin{abstract}
In 60 children with autism to test subjects, borrowing ergonomics in the systematic study of children with autism - Interior color brightness - healing environment relationship, by contrast interior color and brightness to test children with autism to vision exhibit emotional perception changes. The results showed that: bright colors, plenty of brightness allow children with autism early to maintain a pleasant interior, relaxed state, facilitate rehabilitation.
\end{abstract}

\section{Introduction}

Children with autism are a pervasive developmental disorder, at present, China had 3.363 million Children with autism, in the global, and the incidence rate reached five ten thousandths. They live in a very narrow range, language expression and emotional exchanges are varying degrees of problems. The rapid development of social economy did not let this huge number reduced, the gradual improvement of the various mechanisms it has not sought to effective measures in the field of autism, for our country, the treatment of children with autism initiatives still in its infancy. This age group between 3-12 years is the best period of rehabilitation Children with autism. However, the aim of treatment is most autistic children themselves, while ignoring the importance of the environment in the treatment process. Environment on the growth and future development of a person have a vital role, a good environment, can let the children healthy growth, but also can stimulate a child's creativity, imagination and innovation strength ${ }^{[1]}$, good rehabilitation space environment, it can make children get the physical and psychological pleasure and health. So for the lonely children, more should pay attention to the environmental factors. So I think, for the rehabilitation of children with autism research should proceed from environmental factors, how to use color, lighting and other factors create a space environment for children with autism, is a focus in the study of this article.

\section{Methods}

The subjects were 60 Children with autism. $2 \sim 15$ years old autistic children, , children in growth period increasing with age, will be showing a different state. ${ }^{[2]}$ Therefore, we will be divided into three stages Children with autism studied. Early child development period $(2 \sim 5$ years old) 20 people; school-age period (6-12 years old) 20 people; adolescence (12-15 years) 20 people.

In this paper, the instrument measurement method is adopted to test different symptoms of autism children preferred color, brightness, through the display of digital pulse to measure in the face of children with autism have different visual effect has a psychological condition, then through the semantic differential method, namely SD method (Semantic Differential Method) conducted a questionnaire survey ${ }^{[3]}$, using bipolar adjective pair, comfortable and uncomfortable, pleasant and unpleasant, so as to form questionnaire. We use "special anxiety, restlessness, calm, happy, especially happy," again to these numerical statistics, analysis, to obtain the perceived effect of the test population.

Research program is an indoor color Children with autism rehabilitation center, indoor brightness change (Figure 1-2) to evaluate changes in mood autistic children. Indoor color include wall colors, 
furniture and accessories color changes; indoor brightness change including the degree of indoor lighting.

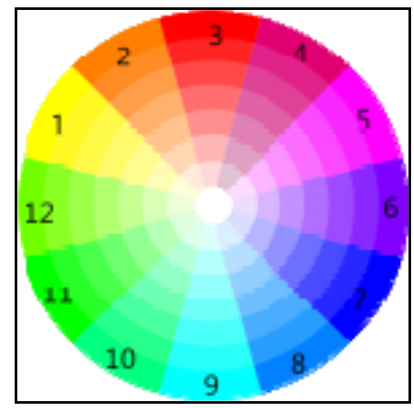

Cool color: 5, 6, 7, 8, 9, 10, warm color: $1,2,3,4,11,12$

Figure 1 distribution of warm and cool colors

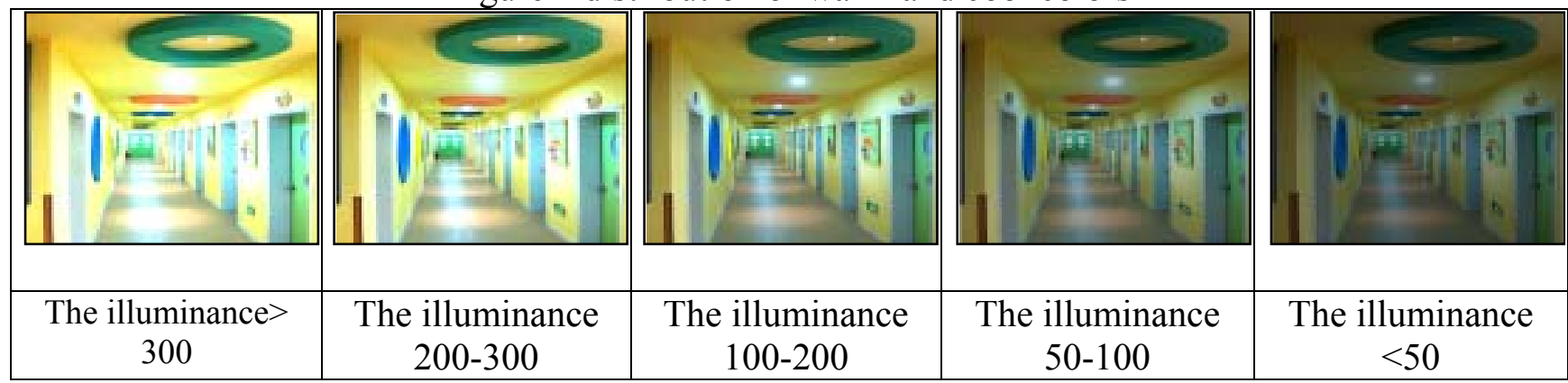

Figure 2 the Interior Design of different intensity illumination under the $5000 \mathrm{~K}$ color temperature

\section{Analyses}

\section{1 environment color and child emotion}

Color as dominant language components, its design language of semantic function is the most prominent in the signifier symbol system. The reasons are as follows: first, color as a visual perception symbol, it brings people to the emotional through perceptual experience, emotional information, which is the kind of semantics; second, a variety of colors has various mental attributes or personality traits are also the same as constitute design language of semanteme information. In the test, the relationship between change color indoor color brightness, hue and purity of both in the space after, the quantity and quality of contrast. The following conclusions:

Survey results show (Figure 3-4), the indoor color changes in test, early childhood development period (2-5 years old) and 20 (hereinafter referred to as Class A); school-age period (6-12 years old) and 20 (hereinafter referred to as Class B); adolescence (12-15 years) and 20 (hereinafter referred to as Class C). Class A, Class B and Class $\mathrm{C}$ test results showed that $70 \%$ of the warm shows, particularly pleasant or unpleasant; $72 \%$ for cool colors showing, anxiety or particularly disturbed.

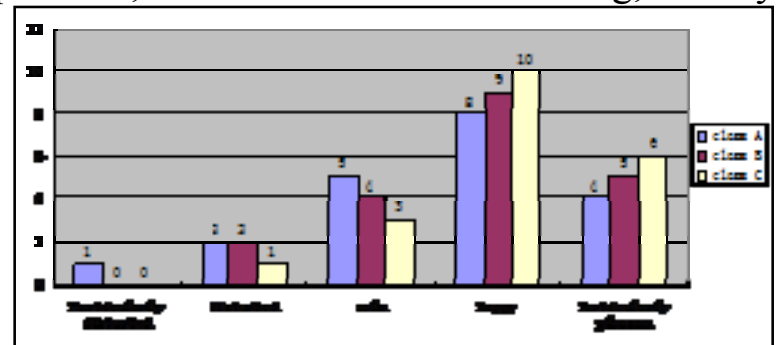

Figure 3.Children's mood changes in the warm color 


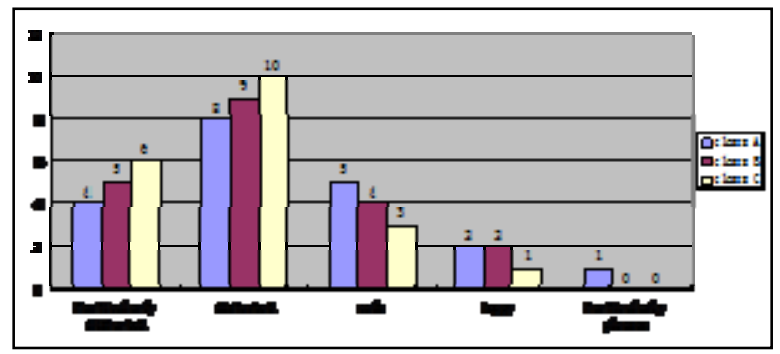

Figure 4. children's mood changes When in the cool color

The remaining 12 people in warm and cool color perception of the change is not strong change, so comprehensive drawn: either Class A, Class B or Class C, on the whole perception of the warm is to make feel good.

\subsection{Ambient brightness and child emotion}

In the rehabilitation of the ambient brightness in the survey, mainly on the relationship between the process of rendering and color temperature between indoor light perceptions were studied and discussed. Among them, about the color temperature, mainly as the standard $5000 \mathrm{~K}(5000 \mathrm{~K}$ as white light, light itself has no shade deviation in this color temperature, showing objects illuminated object qualities), color temperature is the human eye to light emitter or white reflectors, which is physics.

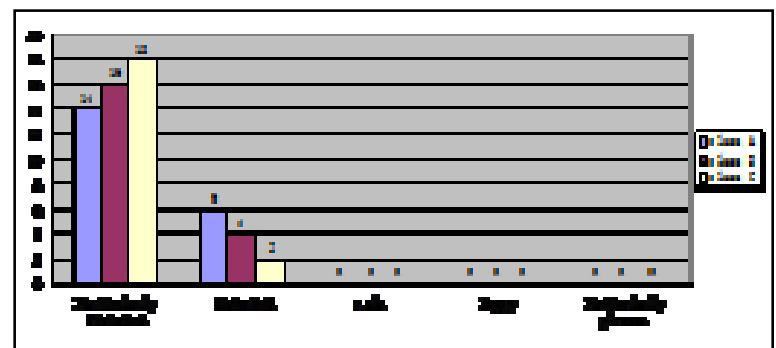

Figure 5. Children's mood changes when the illumination $>300$

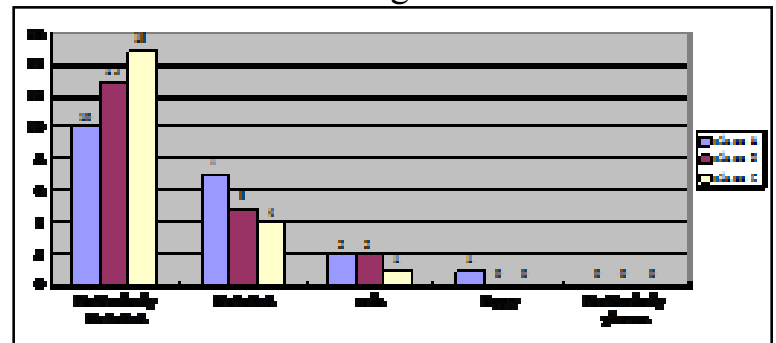

Figure 6. Children's mood changes when the illuminance 200-300

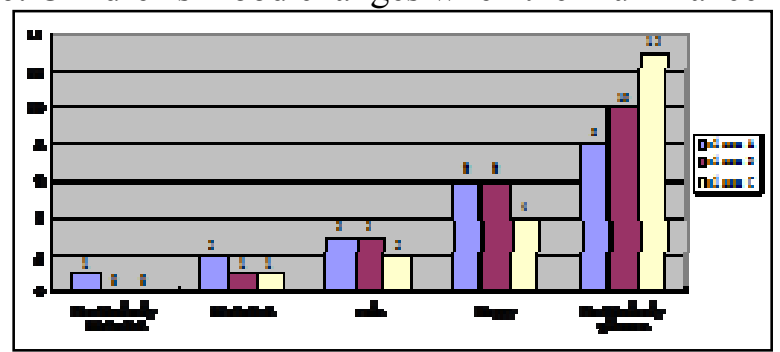

Figure 7. Children's mood changes when illumination 100-200

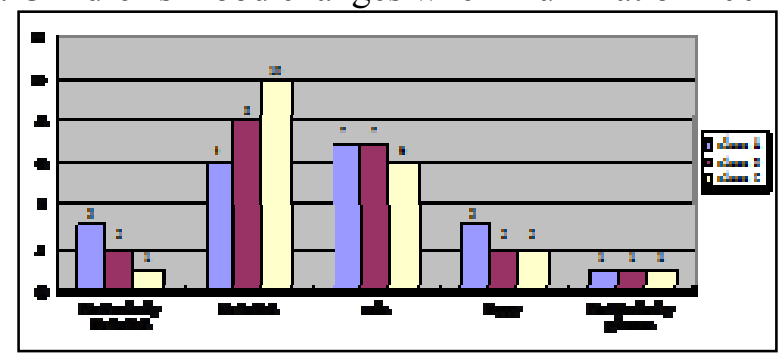

Fgure 8. Children's mood changes when the illuminance $\quad 50-100$ 


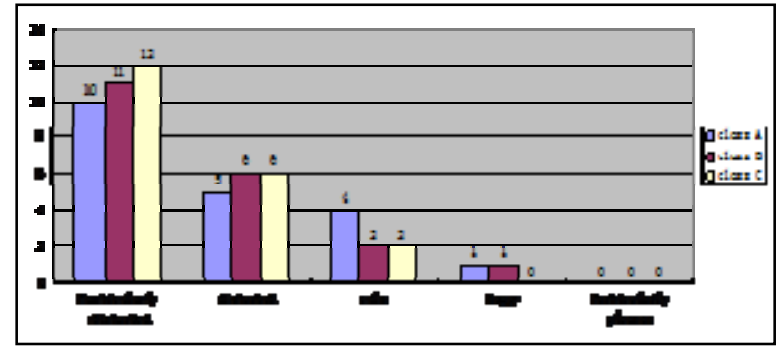

Figure 9. Children's mood changes when the illuminance $<50$

When the survey results show (Figure 5-9), when the illumination is higher than 300, a full three autistic children exhibit anxiety or particularly disturbed state, to reduce illumination to between 200-300, the situation improved somewhat uneasy, there are five people appeared calm state. Continue to reduce the intensity of illumination; the numerical transferred to between 100 and 200, $78 \%$ of people under the illumination presents a pleasure or special pleasant state. When the illumination is 50-100, anxiety and calm the majority; when the illumination is less than $50,83 \%$ of people feel uneasy and particularly disturbed. Transverse and longitudinal comparison: presents the two extremes, intensity of illumination is too high or too low makes people sick. Illumination is most appropriate between 100 and 200.

\section{Conclusions}

In summary, (1) color: compare the cool color, warm colors are more likely to be autistic children receive; when the indoor color temperature of $5000 \mathrm{~K}$, illumination is between 100-200, the brightness in the room at this time most children with autism feel comfortable; therefore, when designing autistic children indoor visual environment system shall comply with the above three points.

\section{Design Recommendations}

1) Adequate lighting. Children's room lamps which choose to reflect in a warm space, lively atmosphere. Adequate shade and enough lighting, so that the room becomes warm, have a sense of security; help eliminate children's fear ${ }^{[4-5]}$. In the light of choice, we try to keep the soft, comfortable, on the exterior lamps to be fun and give children unlimited imagination ${ }^{[6]}$.

2) Bright color. Color for children shape the character played an important role in the delicate [7-8], children's room with colors and patterns must be quite particular about. Color to bright, beautiful, people looked relaxed, pleasant and avoid letting children fall into the dull feeling depressed.

\section{Acknowledgements}

This work was financially supported by "the Ministry of Education Research of Humanities and Social Sciences Funded Projects in China (15YJA760018) (Study on interior and outdoor of environment design of Autistic Children's rehabilitation agency based on ergonomics: Model and Empirical) (2015).

\section{Reference}

[1] Yufang $\mathrm{Xu}$. "Optimizing the kindergarten environment and promoting the development of infant mental health" [J]. "Examination Week", 2012 (70)

[2] The state administrative management papers. "Advances in treatment of children with autism Review" [J] .2011, 5

[3] Jinxia Cheng. Based on the subjective perception of ergonomics research [J], 
[4] Congchao Lu. Tianjin autistic children's health status and intervention trial research [D]. Tianjin: Tianjin Medical University, 2008

[5] Xiao Cai. "Talking about the children's room design principles and elements" [J]. "Chinese Times (in the ten-day) ", 2014.3

[6] Shengxia Zhou. Haiyan Zhou. "Children's room design safety factors" [J]. "China Chemical Industry Trade", 2012.5

[7] Xiaowei Yu. Yun Gao. Tailang Ma. Dechao Liang. Yang Mengyuan. Zhongyi Shi. "The preliminary design movement of temperament toys for Children with autism" [J]. "Science Times", 2013.1

[8] Xunda Gai. "Preschoolers color psychology research" [D]. Tianjin Polytechnic University, 2009 